\title{
Properties and Structure of High Frequency Soft Magnetic Nano-composite Films
}

\author{
Shigehiro Ohnuma ${ }^{1,2 *}$ and Hiroshi Masumoto ${ }^{2}$ \\ ${ }^{1}$ Research Institute for Electric and Magnetic Materials, Sendai 982-0807, Japan \\ ${ }^{2}$ Center for Interdisciplinary Research, Tohoku University, Sendai 980-8578, Japan
}

(Received 2 August 2011, Received in final form 6 September 2011, Accepted 6 September 2011)

\begin{abstract}
Metal-insulator type, nano-granular soft magnetic films have been reviewed from the viewpoint of high frequency magnetic materials. The formation of nano-granular structure is related to the magnitude of heat of formation of intergranule materials. Variation of the ratio of granule phase to intergranule phase in the film is found to produce various characteristics in the magnetic properties of the film. The HRTEM observation reveals that neighboring granules in the film with above 60 at.\% Co, contact at considerable points and the films show soft magnetic properties which are explainable in terms of the random anisotropy model for nanocrystalline materials. Addition of $\mathrm{Ni}$ group elements in $\mathrm{Co}-\mathrm{O}$ based films enhances their anisotropy field up to $400 \mathrm{Oe}$ and they exhibit excellent frequency response of permeability. Also, large electromagnetic noise suppression effect is demonstrated as one of their potential applications.
\end{abstract}

Keywords : granular structure, soft magnetic properties, high frequency, Co-based films, magnetic suppression effect

\section{Introduction}

Since semiconductor electronic devices and the integration of electronic devices are rapidly progressing toward higher frequencies and consequent downsizing, soft magnetic materials with high magnetic permeability, $\mu^{\prime}$, at the $\mathrm{GHz}$ frequency are required. Magnetic permeability vs. frequency dependence for a thin film can be expressed by Landau-Lifshit's equation taking into account the eddy current effect as a function of saturation magnetic induction, $B_{s}$, magnetic anisotropy field, $H_{k}$, specific electrical resistivity, $\rho$, damping factor, and film thickness [1]. Consequently, an excellent high-frequency soft magnetic film is characterized by simultaneously high $\rho, B_{s}$, and $H_{k}$.

Metal-insulator type, nano-granular, magnetic thin films consist of ferromagnetic metal granule several $\mathrm{nm}$ in diameter and ceramic intergranule material of thickness less than $1 \mathrm{~nm}$, and are expected to show high $\rho$ caused by the structure [2]. Changing the film compositions or alloy systems results in valuable properties (hard magnetic properties, giant magnetostriction, soft magnetic properties and so on) in the films. The preparation method of a soft magnetic film with a nano-granular structure by sputtering, was first reported in 1985 [3]. This soft magnetic film, Fe-B-N, was composed of two amorphous phases of Fe-B and B-N and it possessed both large $B_{s}$ $(10 \mathrm{kG})$ and high $\rho(1000 \mu \Omega \mathrm{cm})$. This study suggested the possibility of developing a new class of materials with soft magnetic properties in the range of $\mathrm{GHz}$ frequency. We have so far been extensively studying nano-granular, soft magnetic (named simply "NGSM", hereafter) films as high frequency materials useful at the GHz range. Such a film has been successfully synthesized by forming Co based, metal-insulator type, nano-granular magnetic thin films [4]. NGSM films were found to satisfy these properties with (Fe, $\mathrm{Co}, \mathrm{Ni}$ )-(Al, $\mathrm{Si}, \mathrm{Zr}, \mathrm{Mg}, \mathrm{RE}$ )-(oxide or nitride) and ( $\mathrm{Fe}, \mathrm{Co})-(\mathrm{Al}, \mathrm{Mg})-$ fluoride systems (RE: rare earth elements) [5]. Thus much attention is now being focused on important key materials for electric devices that necessitate further miniaturization and higher frequency operation.

The purpose of this report is to selectively review the work of metal-insulator type NGSM films performed mainly by our group. Particular emphasis will be placed on the relationship between film composition and key properties for further development in the near future.

\footnotetext{
*Corresponding author: Tel: +81-22-245-8027

Fax:+81-22-245-8031, e-mail: s.ohnuma@denjiken.ne.jp
} 


\section{Film Preparatrion and Structure}

Nano-granular films were prepared by a reactive sputtering process under $\mathrm{Ar}+(\mathrm{O}$ or $\mathrm{N})$ gas using $(\mathrm{Fe}, \mathrm{Co})-(\mathrm{M}=$ $\mathrm{Al}, \mathrm{B}, \mathrm{Mg}, \mathrm{Si}, \mathrm{Zr}, \mathrm{RE})$ alloy targets $(\mathrm{RE}=\mathrm{Gd}, \mathrm{Sm}, \mathrm{Y})$, and by co-sputtering or tandem sputtering under $\mathrm{Ar}$ plasma using $\mathrm{Fe}$, Co or alloy target and M-oxide, -nitride or -fluoride target $(\mathrm{M}=\mathrm{Al}, \mathrm{Mg}$ or $\mathrm{Zr})$. The intergranular phase of M-oxide in the film was introduced by preferential oxidation of $\mathrm{M}$ element during deposition. Such a reactive sputtering method using an alloy target was useful for accurately controlling the concentration of gas element and for obtaining films with homogenous composition. The key to finding suitable M-elements which give nano-granular structure is the difference in the heat of formation, $\Delta H$, of $(\mathrm{Fe}, \mathrm{Co})-(\mathrm{O}, \mathrm{N}$ or $\mathrm{F})$ and $\mathrm{M}-(\mathrm{O}, \mathrm{N}$ or F) compounds. For example, The coercive force, $H_{c}$, and $B_{s}$ of Co-M-O films decrease and increases monotonically, respectively, with increasing $\Delta H$ of the intergranule M-oxide phase [2].

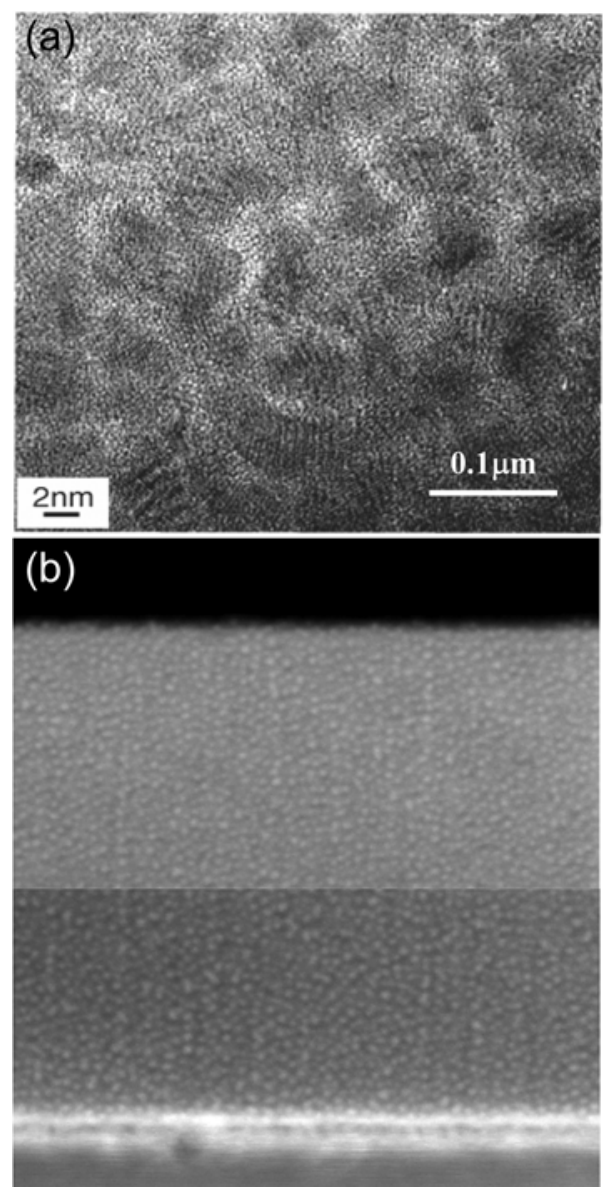

Fig. 1. (a) cross sectional image of $\mathrm{Co}_{69} \mathrm{Al}_{12} \mathrm{O}_{19}$ film by HRTEM, (b) cross sectional view of $\mathrm{Co}_{32} \mathrm{Ta}_{25} \mathrm{O}_{43}$ film by FIBSEM (Vacc. $=1 \mathrm{kV})$.
To clarify the film structure, HRTEM observations were made on Co-Al-O films deposited from a $\mathrm{Co}_{85} \mathrm{Al}_{15}$ target as shown in Fig. 1(a). There can be seen a fairly narrow size distribution of dark granules of several $\mathrm{nm}$ diameter and bright intergranule material. The granules are not completely isolated, and some of them impinge on each other, when the volume fraction of the metallic phase approaches the percolation threshold. One would expect that the granules are magnetically coupled, and a low $H_{c}$ may be caused by the randomly oriented $\mathrm{nm}$ scale Co granules. With decreasing Co content in the films, granules tend to become isolated by the oxide matrix, resulting in the film exhibiting superparamagnetic behaviour [6]. Very recently we have succeeded in observing nano-granular structure Co-Ta-O films by FIB-SEM (Fig. 1(b)) [7]. As can be seen in Fig. 1(b), the granules of nm scale disperse very uniformly from the substrate to the film surface.

Of considerable interest for us is the compositional difference between the granule and the intergranule phases in the film. The composition analysis at nano-scale in the film was examined by means of nm-focused EDX and EELS [2]. By comparing the results for the granules and intergranule regions, a clear difference between the compositions of the two was detected. We found that the amount of $\mathrm{Al}$ element was extremely low in the granules, but high in the intergranule region, while the amount of $\mathrm{O}$ element was high in the intergranule region and low in the granules. On the other hand, the Co concentration was fairly high in the granules. These results of the partial composition analysis were in agreement with our previous expectation of the formation of an Al-O glass close to $\mathrm{Al}_{2} \mathrm{O}_{3}$ as an intergranule phase.

\section{Soft Magnetic Properties}

Since the $H_{c}$ is strongly related to the effective anisotropy, nano-crystalline alloys like Finemet follow Herzer's prediction that the $H_{c}$ should grow as the $6^{\text {th }}$ power of the grain size [8]. The average diameter, $D$, of the granules in NGSM films is almost one order smaller than that of the nano-crystalline materials, and $H_{c}$ increases with increasing $D$ with almost the same tendency as that of nanocrystalline alloys. The relationship between $D$ and $H c$ of NGSM films presents definite evidence for strong exchange interaction among neighboring granules, and can be qualitatively explained by the random anisotropy model.

As mentioned above, higher $\rho, B_{s}$ and $H_{k}$ are the key parameters for excellent high frequency, soft magnetic materials. Since $B_{s}$ is a physical constant and cannot be overcome $2.4 \mathrm{~T}(\mathrm{FeCo})$, the key parameters then become larger $\rho$ and $H_{k}$. In order to obtain a soft magnetic film 


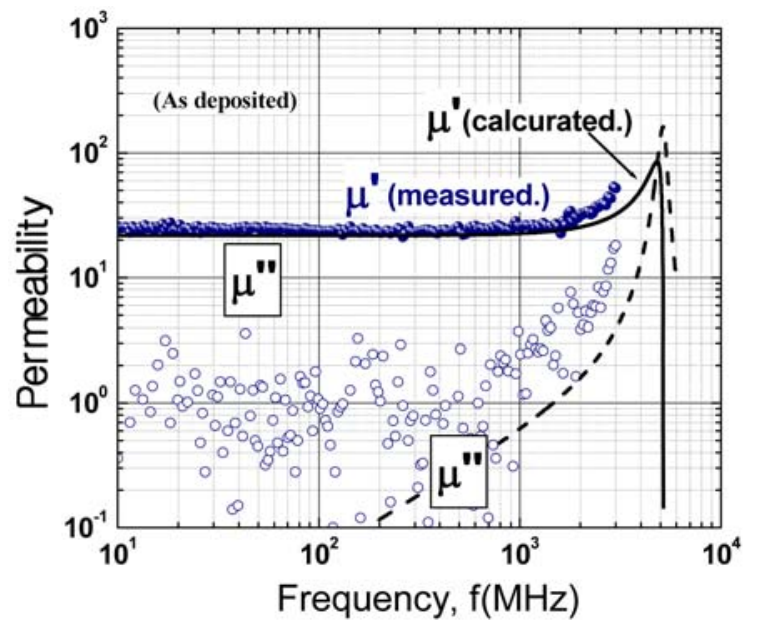

Fig. 2. (Color online) Frequency response of permeability of NGSM Co-Pd-Si-O film.

with larger $H_{k}$ and $\rho$ simultanously, we have focused on Co-based films. Our concept arises from the fact that, generally, Co-based alloys exhibit small magnetostriction and high induced magnetic anisotropy. Therefore, if the granule diameter and the distance between granules (inter-granule region) in the Co-based nano-granular films can be controlled, it may be possible to prepare high frequency soft magnetic films. We have succeeded in preparing several alloy-type NGSM films. For example, soft magnetic Co-Zr-O system films with high $H_{k}$ of more than 100 Oe can be obtained on the wider compositional range than that of Co-Al-O system films [9]. It has been found that the $H_{k}$ of $50-100$ Oe in Co-Al-O films, increases (up to $400 \mathrm{Oe}$ ) by adding $\mathrm{Pt}$, Pd or Ni elements, giving rise to very high frequency, $f$, response of permeability, $\mu$, due to increased resonance frequency, $f_{r}$, as shown in Figure 2. As the result, practical high frequency nano-granular, thin films have been obtained in $\mathrm{Co}-(\mathrm{B}$, or $\mathrm{Si})$-Pd-O films. The most noteworthy properties are $\mu=$ $20, f_{r}=5 \mathrm{GHz}, \rho=5000 \mu \Omega \mathrm{cm}, B_{s}=8 \mathrm{kG}$ and $H_{k}=400$ Oe) [10].

Increments of both $B_{s}$ and $\rho$ in NGSM film can be readily explained by the formation process of granular structure as mentioned above. However, the very large $H_{k}$ cannot be explained by the same reason as for $B_{s}$ and $\rho$. Then we have carried out systematic investigations on field induced, magnetic anisotropy. Measurements were made of the magnetization curves along and across the magnetic easy direction and torque curve [11]. The following properties were found. 1) Torque curve is high of twofold symmetry. 2) The easy direction of magnetization is along the field direction applied during the sputtering or annealing. 3) Uni-axial magnetic anisotropy, $K_{u}$, can be

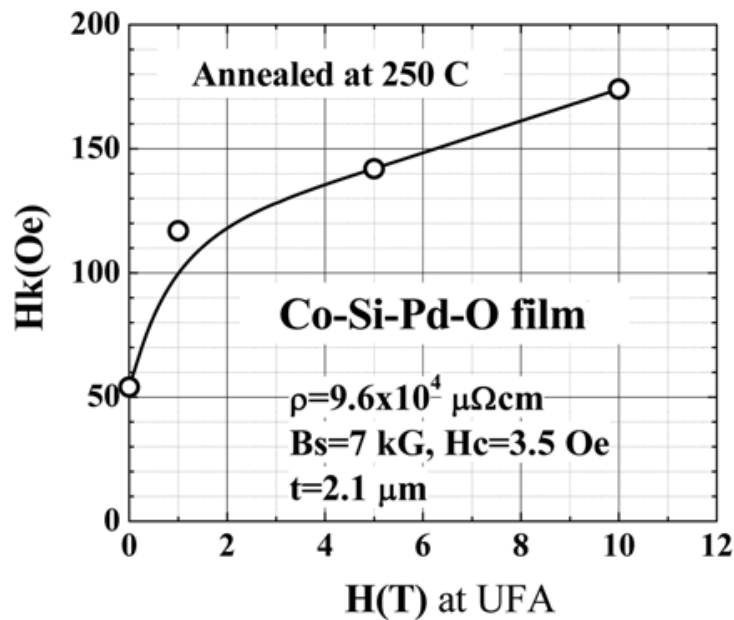

Fig. 3. Magnetic field dependence of magnetic anisotropy field of Co-Pd-Si-O film.

induced reversibly by successive re-annealing in the field. 4) $K_{u}$ depends on the magnetic elements, increasing according to the sequence of Fe based $<\mathrm{Co}$ based $<\mathrm{Co}+\mathrm{Pd}$ based. To confirm this magnetically induced effect, we have tried to apply uni-axial field annealing up to $10 \mathrm{~T}$, keeping temperature constant $\left(250^{\circ} \mathrm{C}\right)$. As can be seen in Fig. 3 , the $H_{k}$ increases with increasing magnetic field from $50 \mathrm{Oe}(0 \mathrm{~T})$ to $170 \mathrm{Oe}(10 \mathrm{~T})$ with small inflection point, which behaves to be dependent upon the film composition. From the above, it is believed that large $K_{u}$ may originate in the $\mathrm{nm}$ scale variation of Co based nanogranular structure. In order to clarify the origin, structural investigations have been performed by means of TEM, small angle X-ray and neutron scattering methods, and will be presented in other paper.

Recently, nitride based Co-Al-N films (Co $\sim 80$ at.\%), when domain structures are controlled, have been found to show a good $\mu-f$ response up to $\mathrm{GHz}$ range, even though they have perpendicular magnetic anisotropy to the film plane [12]. Since the film shows isotropic behavior inplane of the film surface, new applications for high frequency magnetic devices may be developed. On the other hand, Fe based granular films, which are sputter-deposited on the rotating substrate with very fast speed, demonstrate high $H_{k}$ up to 1000 Oe based on the shape anisotropy, and its $f_{r}$ exceeds $5 \mathrm{GHz}$ [13]. Further increment of $K_{s}$ by improving the deposition condition may allows the film $f_{r}$ to reach $10 \mathrm{GHz}$.

\section{Their Applications}

The excellent $\mu-f$ response, therefore, indicates that NGSM films are strong candidates for magnetic cores, 


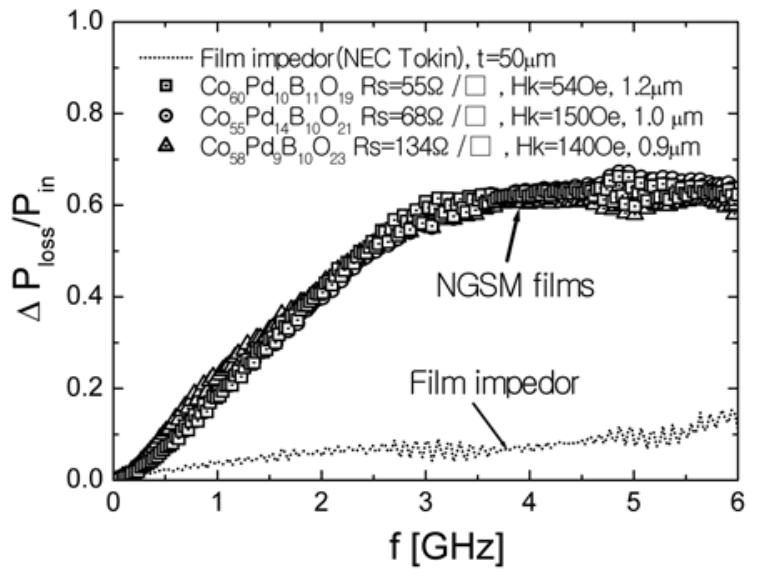

Fig. 4. Frequency vs power loss of various Co-Pd-B-O films (data of commercial noise suppressor is shown for comparison).

such as for inductors and magnetic recording heads at $\mathrm{GHz}$ frequency. These results are worthwhile not only for applications using high $\mu^{\prime}$ and quality factor, but, also, for devices using high $\mu^{\prime \prime}$. Since $\mathrm{Co}_{85} \mathrm{~B}_{15}$-Pd-O NGSM films have large electrical loss and magnetic loss near fr, they are expected, for example, to be useful as an excellent noise suppressor in the semi-micro wave frequency range. A micro strip line, $M S L$, of $Z c=50 \Omega$, whose ends were both connected to a network analyzer, was used to evaluate the noise suppression effect [14].

Fig. 4 shows the frequency dependence of noise suppression effect, $\Delta P_{\text {loss }} / P_{\text {in }}$, up to $6 \mathrm{GHz}$ for several kinds of $\mathrm{Co}_{85} \mathrm{~B}_{15}$-Pd-O films. $\Delta P_{\text {loss }}$ indicates the real power loss of magnetic films, which has been determined by subtracting the MSL part from the total power loss [15]. The result of Film Impedor (NEC-Tokin Co. Japan, $50 \mu \mathrm{m}$ thickness), which is well known as an electromagnetic noise suppression material at high frequency, is also shown in Fig. 4 for comparison. The $\Delta P_{\text {loss }} / P_{\text {in }}$ ratio of the films increases with increasing frequency up to $3 \mathrm{GHz}$ and remains constant (around 0.6) in frequencies higher than $3 \mathrm{GHz}$. It is noted that $\Delta P_{\text {loss }} / P_{\text {in }}$ ratios of the NGMS films are about 6 times larger than that of the Film Impedor despite the thickness of Film Impedor being 50 times larger than those of the NGMS films. The excellent noise suppressing effect of soft magnetic $\mathrm{Co}_{85} \mathrm{~B}_{15}-\mathrm{Pd}-\mathrm{O}$ films is attributed to large electric loss due to a sheet resistance around $100 \Omega$ and a magnetic loss or $\mu^{\prime \prime}$ near $f_{r}$. We have deposited NGSM film directly on the LSI with top cover removed, and found that NGSM films reduced noise level more than $50 \%$. In some applications for noise suppression, the thinness of these films is advantageous for heat removal from electric devices.

\section{Closing Remarks}

Our recent results on nano-granular, magnetic thin films are introduced and discussed from the viewpoint of high frequency, soft magnetic properties. We have shown high frequency noise suppression effect as one of their applications. These results are expected to be important for "information technology" in the near future.

Basic magnetic properties of NGSM films have been also investigated for developing new functional properties in the near future, and several interesting properties attributed to their structure have been found. The $\rho$ of NGSM Co-Al-O films, for example, decreases with lowering temperature and shows a $T^{2}$-behavior in the range 50 $<T<150 \mathrm{~K}$, suggesting the scattering of conduction electrons by thermally excited ferromagnetic spin waves. At temperatures then lower than $50 \mathrm{~K}$, the $\rho$ deviates from $T^{2}$-behavior. It reaches a minimum and the increases logarithmically with decreasing temperature. The $\rho$ begins to deviate from $\log T$-law below $0.5 \mathrm{~K}$ and is almost temperature independent below $100 \mathrm{mK}$. This behavior looks like $\rho$ in the impurity Kondo system [16].

\section{Acknowlegement}

The work reported here has been performed in collaboration with M. Ohnuma, S. Mitani and K. Hono of National Institute for Material Science, M. Yamaguchi, Y. Shimada of Tohoku University, and T. Iwasa, H. J. Lee and N. Kobayashi of Research Institute for Electric and Magnetic Materials, RIEMM. We gratefully acknowledge the valuable comments and continuous encouragement of Prof. H. Masumoto and Prof. H. Hujimori of RIEMM.

This work was supported mainly by a grant from The Japan Society for the Promotion of Science.

\section{References}

[1] A. Hosono and Y. Shimada, J. Magn. Soc. Jpn. 12, 295 (1988).

[2] M. Ohnuma, K. Hono, H. Onodera, S. Ohnuma, H. Fujimori, and J. S. Pedersen, J. Appl. Phys. 85, 817 (2000).

[3] H. Karamon, T. Masumoto, and Y. Makino, J. Appl. Phys. 57, 3527 (2000).

[4] S. Ohnuma, H. Fujimori, S. Mitani, and T. Masumoto, J. Appl. Phys. 79, 5130 (1996).

[5] N. Kobayashi, S. Ohnuma, T. Masumoto, and H. Fujimori, J. Magn. Soc. Jpn. 22, 581 (1998).

[6] H. Fujimori, S. Mitani, and S. Ohnuma, Mater. Sci. Eng. B31, 219 (1995).

[7] S. Ohnuma, T. Iwasa, K. Tanaka, K. Man, and H. Masumoto, Abstract of Fall Meeting of Japan Metal Society 
(2010) pp. 344.

[8] G. Herzer, IEEE Trans. Magn. 25, 3327 (1989).

[9] S. Ohnuma, H. J. Lee, N. Kobayashi, H. Fujimori, and T. Masumoto, IEEE Trans. Magn. 37, 2251 (2001).

[10] S. Ohnuma, N. Kobayashi, T. Masumoto, S. Mitani, and H. Fujimori, J. Magn. Soc. Jpn. 23, 240 (1999).

[11] H. J. Lee, S. Ohnuma, H. Fujimori, and T. Masumoto, J. Magn. Soc. Jpn. 24, 687 (2000).

[12] H. Kijima, S. Ohnuma, and H. Masumoto, IEEE Trans. Magn. 47, 3928 (2001).
[13] M. Munakata, S. I. Aoqui, and M. Yagi, IEEE Trans. Magn. 41, 3262 (2005).

[14] S. Yoshida, H. Ono, S. Ando, F. Tsuda, T. Ito, Y. Shimada, M. Yamaguchi, K. I. Arai, S. Ohnuma, and T. Masumoto, IEEE Trans. Magn. 37, 2401 (2001).

[15] S. Ohnuma, H. Nagura, H. Fujimori, and T. Masumoto, IEEE Trans. Magn. 40, 2712 (2004).

[16] S. Nakamura, T. Nojima, A, Yoshihara, S. Ohnuma, and H. Fujimori, J. Phys. Soc. Jpn. 78, 074708 (2009). 